\section{DmGTSF1 is necessary for Piwi-piRISC-mediated transcriptional transposon silencing in the Drosophila ovary}

\author{
Hitoshi Ohtani, ${ }^{1}$ Yuka W. Iwasaki, ${ }^{1}$ Aoi Shibuya, ${ }^{1}$ \\ Haruhiko Siomi, ${ }^{1}$ Mikiko C. Siomi, ${ }^{2,3}$ \\ and Kuniaki Saito ${ }^{1,3}$
}

${ }^{1}$ Department of Molecular Biology, Keio University School of Medicine, Tokyo 160-8582, Japan; ${ }^{2}$ Department of Biophysics and Biochemistry, Graduate School of Science,

The University of Tokyo, Tokyo 113-0032, Japan

The Piwi-piRNA (PIWI-interacting RNA) complex (PiwipiRISC) in Drosophila ovarian somatic cells represses transposons transcriptionally to maintain genome integrity; however, the underlying mechanisms remain obscure. Here, we reveal that DmGTSF1, a Drosophila homolog of gametocyte-specific factor 1 (GTSF1) (which is required for transposon silencing in mouse testes), is necessary for Piwi-piRISC to repress target transposons and neighboring genes. DmGTSF1 depletion affected neither piRNA biogenesis nor nuclear import of PiwipiRISC. DmGTSF1 mutations caused derepression of transposons and loss of ovary follicle layers, resulting in female infertility. We suggest that DmGTSF1, a nuclear Piwi interactor, is an integral factor in Piwi-piRISCmediated transcriptional silencing.

Supplemental material is available for this article.

Received May 8, 2013; revised version accepted June 26, 2013.

Mobilization of transposable elements (TEs) can lead to natural insertion mutations that generally have negative effects on the host genome (Lynch 2007). Thus, host species have evolved control mechanisms that restrict TE activity. One such mechanism is RNAi/RNA silencing, in which small RNAs 20-30 nucleotides (nt) in length trigger multiple forms of sequence-specific gene silencing by guiding Argonaute complexes or RNA-induced silencing complexes (RISCs) to target RNAs by means of basepairing (Ketting et al. 1999; Tabara et al. 1999; Slotkin and Martienssen 2007; Siomi and Siomi 2009).

In the Drosophila germline, PIWI-interacting RNAs (piRNAs) are produced from single-stranded long noncoding RNAs that are transcribed from intergenic piRNA clusters on the genome. Mature piRNAs are loaded onto germline-specific Argonaute proteins or PIWI proteins to

[Keywords: DmGTSF1; piRNA; Piwi; transposon; RNA silencing; Drosophila] ${ }^{3}$ Corresponding authors

E-mail saito@z6.keio.jp

E-mail siomim@biochem.s.u-tokyo.ac.jp

Article is online at http://www.genesdev.org/cgi/doi/10.1101/gad.221515.113. yield piRISCs. piRNAs are amplified by the cytoplasmic "ping-pong" cycle, in which TE transcripts are consumed as both the source of piRNAs and the targets of PIWI cleavage, thereby repressing TEs in the cytoplasm (Karginov and Hannon 2010; Senti and Brennecke 2010; Siomi et al. 2011). It is becoming clear that piRISCs also mediate TE silencing in the nucleus but in a PIWI cleavage-independent manner (Saito et al. 2010; Klenov et al. 2011; Wang and Elgin 2011; Sienski et al. 2012; Darricarrère et al. 2013).

Although three PIWI proteins-AGO3, Aubergine (Aub), and Piwi-are expressed in the Drosophila ovary (Brennecke et al. 2007), only Piwi is expressed in ovarian somatic cells (OSCs), such as follicle cells, where piRNA intermediates are digested by a single-stranded nucleic acid-specific endonuclease, Zucchini (Ipsaro et al. 2012; Nishimasu et al. 2012). Zucchini products are further processed in the cytoplasmic $\mathrm{Yb}$ bodies into mature piRNAs /Olivieri et al. 2010; Saito et al. 2010). Piwi-piRISC formation also takes place at $\mathrm{Yb}$ bodies, where several piRNA biogenesis factors, including Armitage (Armi), accumulate (Olivieri et al. 2010; Saito et al. 2010). piRNAs in OSCs are not amplified because these cells lack the core factors (i.e., AGO3 and Aub). Piwi-piRISCs are imported into the nucleus, where they mediate TE repression. Nuclear silencing by Piwi-piRISCs does not require cleavage of TE transcripts but involves chromatin modification of TE loci on the genome (Saito et al. 2010; Wang and Elgin 2011; Sienski et al. 2012; Huang et al. 2013; Le Thomas et al. 2013; Rozhkov et al. 2013). However, the underlying mechanism remains completely unknown.

To identify genes required for the TE silencing mediated by Piwi-piRISCs, we used a candidate approach. An earlier study showed that mouse gametocyte-specific factor 1 (GTSF1) is required for TE silencing in the testis (Yoshimura et al. 2009). GTSF1 knockout male mice show infertility owing to a lack of both DNA methylation and subsequent TE silencing during meiosis in spermatocytes, phenocopying mice lacking Mili or Miwi2 (Yoshimura et al. 2007, 2009; Aravin et al. 2008; Kuramochi-Miyagawa et al. 2008; Pillai and Chuma 2012), both of which encode mouse PIWI proteins. In the present study, we knocked down four homologs of GTSF1 in a cultured Drosophila OSC line (Saito et al. 2009) and found that depletion of one particular homolog, CG3893, results in derepression of TEs and neighboring genes, although the level and localization of Piwi-piRISCs were unchanged. We term CG3893 as DmGTSF1 hereafter. DmGTSF1 is a nuclear protein that interacts with Piwi, and a lack of DmGTSF1 results in strong enrichment of RNA polymerase II (Pol II) and less efficient enrichment of trimethylated histone H3 Lys 9 (H3K9me3) at TE loci on the genome, suggesting that DmGTSF1 is an integral factor in Piwi-piRISC-mediated transcriptional silencing.

\section{Results and Discussion}

OSCs were transfected with individual siRNAs against DmGTSF1 and its paralogs, CG14036, CG32625, and CG34283 (Supplemental Fig. S1A), and Northern blotting and RT-qPCR were performed to examine the levels of piRNAs and mdg1 transcripts, respectively, in the transfected cells. None of the siRNAs changed the expression 
levels of piRNAs, Idefix-piR-1, and/or traffic jam-piR-1 (Fig. 1A; Supplemental Fig. S1B). However, knockdown of DmGTSF1, but not of its paralogs, increased the level of mdg1 (Fig. 1B; Supplemental Fig. S1C), a long terminal repeat element, suggesting that only DmGTSF1 is required for TE silencing. Depletion of Maelstrom (Mael), a known piRNA factor (Lim and Kai 2007), led to almost identical phenotypes (Fig. 1A,B). A recent study showed that Mael is required for transcriptional TE silencing mediated by Piwi-piRISC but not for piRNA biogenesis (Sienski et al. 2012), suggesting the existence of functional parallels between Mael and DmGTSF1. Immunofluorescence staining for Myc-DmGTSF1 in OSCs revealed that DmGTSF1 shows a nuclear localization, as does Piwi (Fig. 1C). This further supports the idea that DmGTSF1 functions in nuclear TE silencing. Depletion of HP1a derepressed mdg1 (Fig. 1B), consistent with the earlier observation that HP1a functions in TE silencing in the ovaries (Wang and Elgin 2011). The expression levels of piRNAs were unaffected by a lack of HP1a (Fig. 1A; Saito et al. 2010). Thus, piRNA biogenesis does not require HP1a. Loss of Armi, a piRNA biogenesis factor (Olivieri et al. 2010; Saito et al. 2010), resulted in down-regulation of piRNAs, verifying our results.

To investigate the roles of DmGTSF1 in fertility, females of the P-element insertion line $P\{w[+m C]=G S V 6\} G S 12962$ (Supplemental Fig. S2A), in which DmGTSF1 mRNA and its protein product are expressed below detection levels (Fig. 2A; Supplemental Fig. S2B), were crossed with Oregon $R$ male flies. DmGTSF1 ${ }^{-1-}$ females laid no eggs (data not shown). In contrast, females in the reciprocal cross laid eggs (data not shown). The DmGTSF1 transcript was barely detected in testes (Graveley et al. 2011), and
A

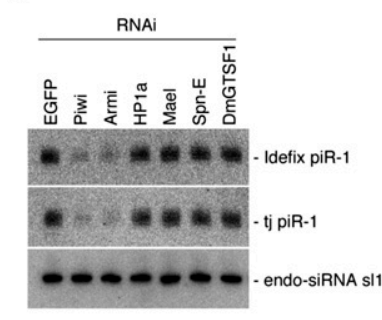

C

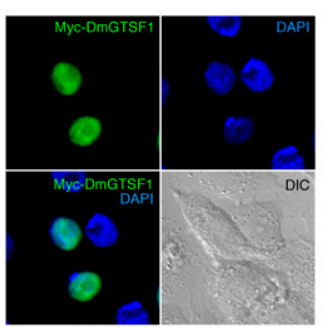

B

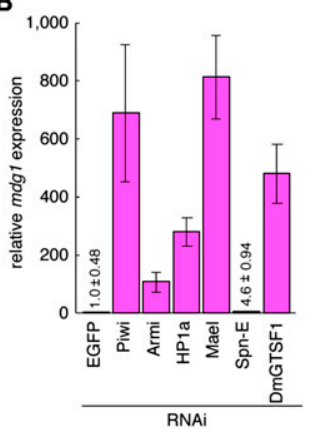

Figure 1. DmGTSF1, a nuclear factor, is necessary for TE silencing but not piRNA biogenesis in OSCs. (A) DmGTSF1 and with the known piRNA factors Piwi, Armi, HPla, Mael, and Spn-E were depleted in OSCs by RNAi and Northern blotting performed on total RNAs isolated from the cells $48 \mathrm{~h}$ post-transfection. The levels of Idefix-piRNA and ti-piRNA were unchanged by DmGTSF1 depletion, suggesting the dispensability of DmGTSF1 in piRNA biogenesis in OSCs. $(B)$ RT-qPCR showed that $m d g 1$ is derepressed by DmGTSF1 depletion, as it is by Piwi depletion. (C) Immunofluorescence for Myc-DmGTSF1 (green) in OSCs. DAPI staining (blue) shows the locations of the nuclei.
DmGTSF1 ${ }^{-/-}$testes appeared normal (data not shown). Thus, DmGTSF1 is specifically required for female fertility.

DMGTSF1 ${ }^{-/-}$ovaries appeared rather small and severely deformed, similar to those observed in Piwi mutants (Lin and Spradling 1997). A comparison of Aub immunostaining images with images showing DAPI-stained nuclei revealed that DmGTSF1 ${ }^{-1-}$ ovaries lack a follicle cell layer, which encapsulates each egg chamber in DmGTSF1 $1^{+-}$ovaries (Fig. 2B).

Immunostaining was also performed using antibodies against the polar follicle cell marker FasIII (Ruohola et al. 1991) and the oocyte marker protein Orb (Christerson and McKearin 1994). In DmGTSF1 ${ }^{+/-}$ovaries, FasIII is detected in monolayered follicle cells in both the germarium and early stage egg chambers, but later, its expression becomes restricted to two polar follicle cells in individual egg chambers, as has been observed in wild-type ovaries (Fig. 2C; Ruohola et al. 1991). However, in DmGTSF1-/ovaries, monolayered FasIII-positive cells were lost at early stages; rather, FasIII-positive cells were dispersed within the germarium-like structures. Mutant egg chambers at later stages contained multiple FasIII-positive cells (Fig. 2C).Polar cell specification is likely disrupted owing to the loss of DmGTSF1.

Orb expression is normally activated during mitotic division. Then, Orb gradually accumulates in oocytes during development (Christerson and McKearin 1994). This spatiotemporal expression pattern of Orb was observed in DmGTSF $1^{+/-}$ovaries (Fig. 2D). However, in DmGTSF1 ${ }^{-/-}$ovaries, Orb appeared in all cells within the early cysts, and its expression was seen in multiple cells in late egg chambers (Fig. 2D). These DmGTSF1 mutant phenotypes are very similar, if not identical, to those of piRNA pathway mutants: The number of follicle cells was also reduced in the ovaries of mutant $f_{S}(1) Y b$, a piRNA biogenesis factor and a key component of $\mathrm{Yb}$ bodies (Szakmary et al. 2009; Olivieri et al. 2010; Saito et al. 2010). Fusion of egg chambers caused by loss of Mael and $\mathrm{fs}(1) \mathrm{Yb}$ also resulted in aberrant multiplicity of Orbpositive cells in late egg chambers (Johnson et al. 1995; Sato et al. 2011). These overlapping phenotypes further suggest functional parallels between Mael and DmGTSF1.

DmGTSF1 mutant flies appear otherwise normal. This correlates well with the observation that a subset of TEs was derepressed in DmGTSF1 ${ }^{-1-}$ ovaries but not in the carcasses of DmGTSF1 ${ }^{-1-}$ flies (Supplemental Fig. S2C). The RT-qPCR results confirm the requirement of DmGTSF1 for TE silencing not only in OSCs, but also in the ovaries.

To compare the levels of transcripts globally, we performed mRNA sequence (mRNA-seq) analysis of OSCs transfected with siRNAs against the known piRNA pathway genes Piwi, Mael, and Armi along with DmGTSF1 and normalized the expression levels to those in OSCs transfected with control siRNAs against EGFP. mRNA-seq samples were prepared at $48 \mathrm{~h}$ post-transfection, yielding $168,369,636$ genome-mapped reads in total (Supplemental Table S1). Reads per kilobase per million mapped reads (RPKM) values for annotated genes were highly correlated between Piwi knockdown and control knockdown cells $\left(R^{2}=0.99\right)$. In contrast, several of the 125 annotated Drosophila melanogaster TE families showed strong increases in RNA levels (Fig. 3A,B). For example, the transcript levels for the long terminal repeat elements mdg1, gypsy, and 297 were increased by $>156$-fold, $>32$ fold, and >17-fold, respectively, upon Piwi knockdown, 
A

\section{C}
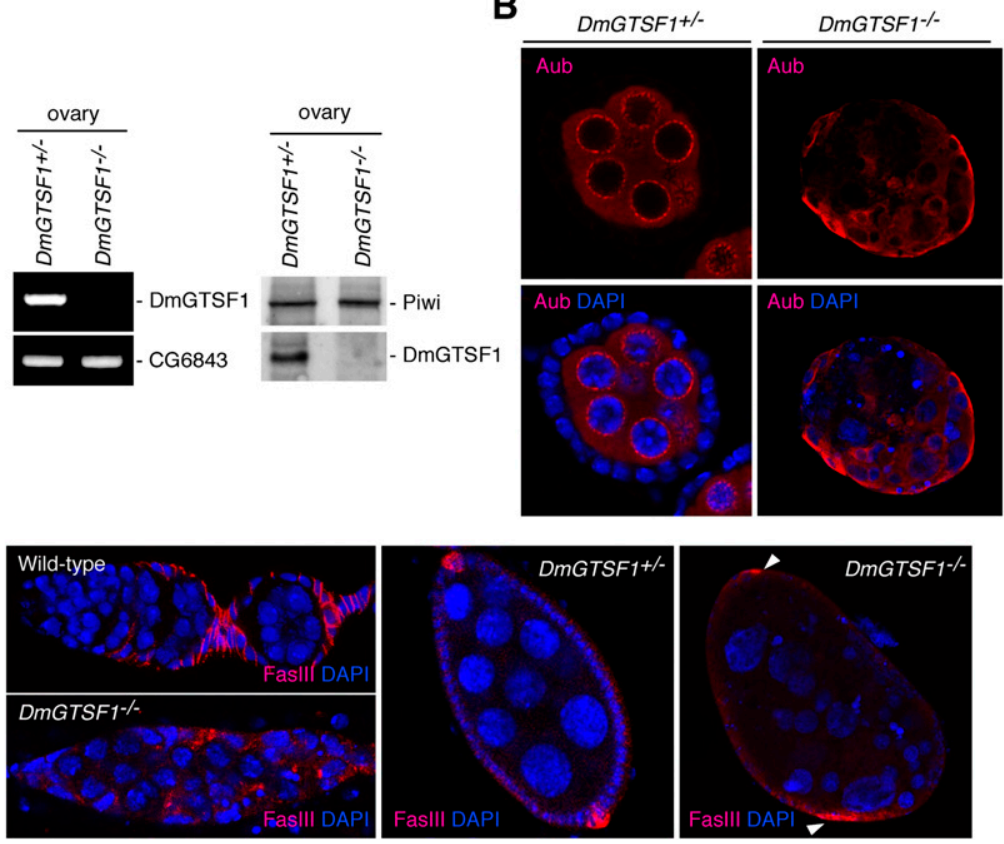

D

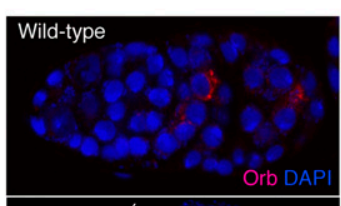

DmGTSF1\%
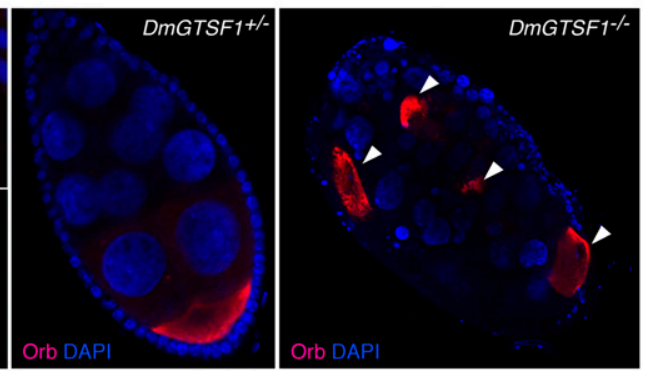

Figure 2. DmGTSF1 is essential for development of the ovaries. $(A, l e f t)$ RT-PCR results showing that the homozygous $P\{w[+m C]=G S V 6\} G S 12962$ mutant does not express DmGTSF1 mRNAs but does express CG6843, a neighboring gene. A schematic drawing of DmGTSF1 (green) and CG6843 (magenta) genes on the Drosophila genome is shown in Supplemental Figure S2A. $+1-1 \quad P\{w[+m C]=G S V 6\} G S 12962 / T M 3$; $1-1-1$ $P\{w[+m C]=G S V 6\} G S 12962 / P\{w[+m C]=G S V 6\} G S 12962$. (Right) Western blot showing that DmGTSF1 $1^{-1-}$ flies do not express DmGTSF1 protein but do express Piwi. A monoclonal antibody against DmGTSF1 was generated, and the specificity is shown in Supplemental Figure S2B. ( $B$, left) Immunostaining images showing that Aub (red) is expressed only in germline cells that are encapsulated by somatic follicle cells, which are positive by DAPI-staining in DmGTSF1 ${ }^{+/-}$flies. (Right) In contrast, the DmGTSF1 egg chamber lacks follicular epithelium morphogenesis. (C) Polar cell specification is defective in DmGTSF1 $1^{-1-}$ ovaries. (Left) Ovaries were immunostained with antibodies against a polar follicle cell marker, FasIII (red). Enlarged images of the DmGTSF1 ${ }^{+-}$egg chamber (middle) and the DmGTSF1 ${ }^{-1-}$ egg chamber (right) are shown. FasIII-positive cells in the mutant are indicated by white arrowheads. $(D)$ Oocyte polarity and specification are defective in DmGTSF1 ${ }^{-1-}$ ovaries. (Left) Ovaries were immunostained with antibodies against an oocyte marker protein, Orb (red). Enlarged images of the DmGTSF1 ${ }^{+/-}$egg chamber (middle) and the DmGTSF1 ${ }^{-/-}$egg chamber (right) are shown. Several Orb-positive cells are detected in the DMGTSF1 $1^{-/-}$ovary (arrowheads).

supporting the recent results of Sienski et al. (2012). Almost identical results were obtained upon knockdown of Armi, Mael, and DmGTSF1 (Fig. 3B; Supplemental Fig. S3B). This suggests that not only Armi (Haase et al. 2010; Olivieri et al. 2010; Saito et al. 2010) and Mael (Sienski et al. 2012) but also DmGTSF1 function in the Piwi-piRISC pathway in OSCs. RT-qPCR confirmed that $m d g 1$, but not other TEs such as I-element and Het-A, was markedly derepressed in Piwi- and DmGTSF1-depleted cells (Supplemental Fig. S3A). Although HP1a is required for silenc- ing of various TEs in OSCs (Fig. 3B), the TE derepression pattern was rather different in HP1a-depleted cells $\left(R^{2}=0.49\right)$ (Supplemental Fig. S3B). Depletion of DmGTSF1 does not affect the levels or cellular localization of Piwi and Mael in OSCs (Supplemental Fig. S3C,D; data not shown), confirming that the observed defective phenotypes were not due to a secondary effect of DmGTSF1 depletion. Likewise, loss of Mael and HPla did not change the cellular localization of DmGTSF1 (Supplemental Fig. S3E).

Piwi-piRISCs influence the expression of protein-coding genes near TE insertions (Sienski et al. 2012). To assess the role of DmGTSF1 in this effect, we analyzed our mRNA-seq data for 28 genes shown in the previous study (Fig. 3C,D; Sienski et al. 2012). As expected, the expression levels of these neighboring genes were comparable between DmGTSF1 and Piwi knockdown cells. The results for some genes (for example, bab1 and CG12477) did not coincide with those of the previous study (Sienski et al. 2012), although transposon insertions near the genes were confirmed by PCR (Supplemental Fig. S4). This variegation may be due to subtle unexpected differences in knockdown and/or cell manipulation conditions. Collectively, all of these bioinformatic data strongly support the notion that DmGTSF1 is an integral factor in the Piwi-piRNA pathway.

Both Piwi and DmGTSF1 are localized to the nucleus (Fig. 1C; Supplemental Fig. S3D). We then asked whether DmGTSF1 forms a complex with Piwi in vivo. Myc-DmGTSF1 fused C-terminally with EGFP (Myc-DmGTSF1EGFP) was expressed in OSCs, and the complex immunoprecipitated with an anti-Myc antibody was probed with an anti-Piwi antibody. Myc-DmGTSF1-EGFP and endogenous Piwi coprecipitated (Fig. 4A) even in the presence of RNase A (Supplemental Fig. S5A).

An in vitro pull-down assay using recombinant DmGTSF1 fused to glutathione S-transferase (GST-DmGTSF1) and crude OSC lysates confirmed their association (Fig. 4B; Supplemental Fig. S5B): Piwi was detected with GSTDmGTSF1 to an extent similar to that with GST-Pimet/DmHen1, which interacts with Piwi and 2'-O-methylates piRNAs upon Piwi loading (Horwich et al. 2007; Saito et al. 2007). Under the conditions we used, Mael was not detected in the DmGTSF1 complex (Supplemental Fig. S5C), although endogenous Mael was partially localized to the nucleus (Supplemental Fig. S5D). Moreover, neither Armi nor AGO1, another Argonaute protein and a component of the microRNA pathway in Drosophila (Okamura et al. 2004), was detected with GST-DmGTSF1 (Fig. 4B). Armi is cytoplasmic and interacts with Piwi (Olivieri et al. 2010; Saito et al. 2010), suggesting that Piwi changes protein interactors upon nuclear import.

DmGTSF1 consists of 167 amino acid residues and contains two U11-48K-like CHHC-type Zn finger motifs 
A
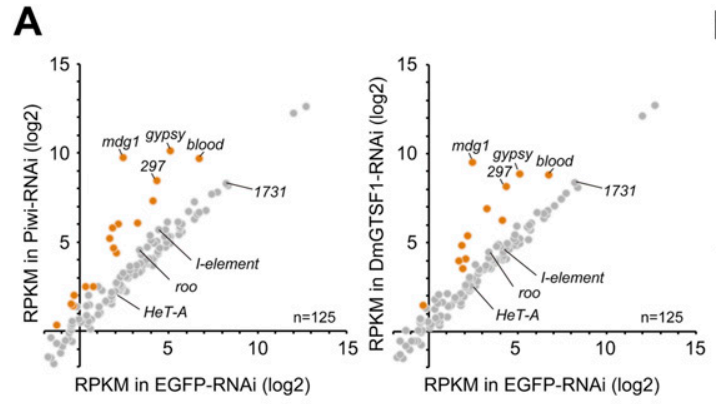

C

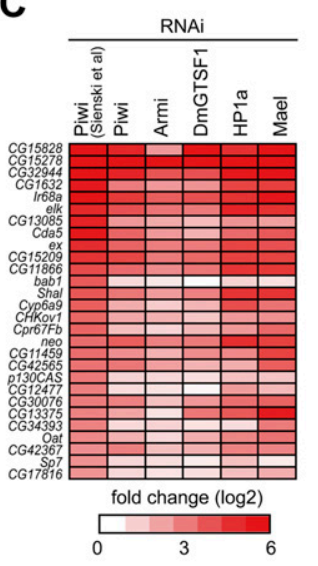

D

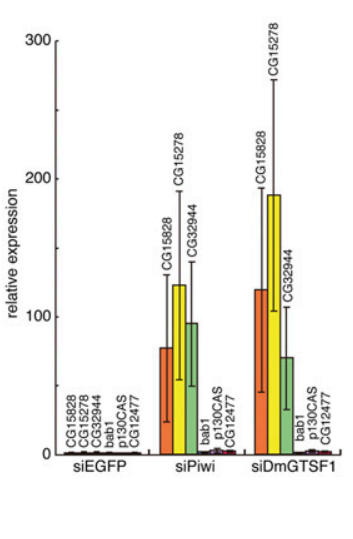

B

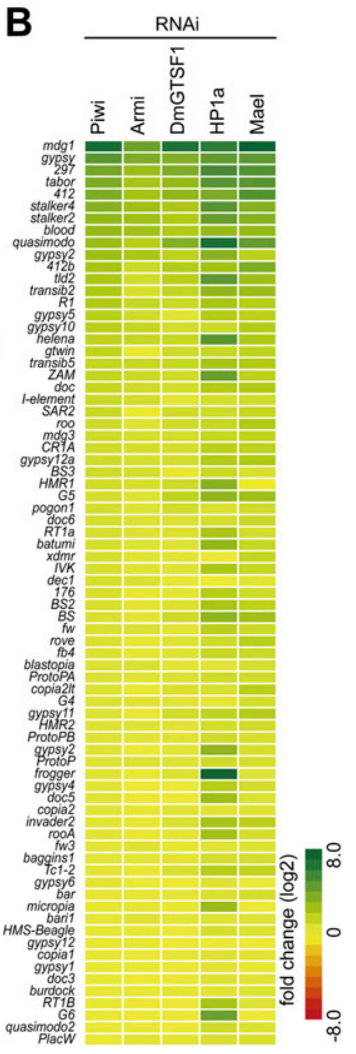

Figure 3. DmGTSF1 regulates expression of TEs and their neighboring genes in the Piwi pathway. (A) Scatter plot of RPKM values (log2) for TEs in EGFP (control) or Piwi (left)/ DmGTSF1 (right) RNAi knockdown samples based on RNA sequencing (RNA-seq). TEs whose expression level differs from that in the control by more than threefold are colored orange. (B) Fold change of TEs to EGFP (control) RNAi knockdown are displayed as heat maps for Piwi, Armi, DmGTSF1, HP1a, and Mael RNAi knockdown samples. $(C)$ Heat maps indicating the fold change of 28 genes, which are mapped TE insertions in either the gene body or close proximity (up to $5 \mathrm{~kb}$ away) and up-regulated in Piwi RNAi knockdown cells (Sienski et al. 2012). Fold change of these genes in Piwi, Armi, DmGTSF1, HP1a, and Mael RNAi knockdown samples. (D) The results of mRNA-seq analyses are recapitulated by RT-qPCR analysis. The steady-state levels of transcripts from protein-coding genes (as described in C) were measured relative to RP49.

(Andreeva and Tidow 2008) in which four cysteines (C4 and C24 in the first domain; C37 and C57 in the second domain) are highly conserved among orthologs and paralogs of DmGTSF1 (Supplemental Fig. S1A). We mutated these four cysteines individually to alanine and asked whether the mutations would abolish the function of DmGTSF1. We conducted rescue experiments in which endogenous DmGTSF1 was depleted by RNAi, and RNAiresistant DmGTSF1 wild type (DmGTSF1r) and DmGTSF1 mutants were individually expressed in DmGTSF1-depleted OSCs. RT-qPCR showed that mutations in the $\mathrm{Zn}$ finger motifs decreased TE silencing activity (Fig. 4C). All of the DmGTSF1 mutants localized to the nucleus (Supplemental Fig. S6A; data not shown) and associated with Piwi (Supplemental Fig. S6B), showing that functional Zn finger motifs are not required for the interaction with Piwi. This also implies that the interaction of DmGTSF1 with Piwi is not sufficient for retrotransposon silencing and that the Zn fingers have a crucial role in this process through as yet unidentified factors.

We next performed chromatin immunoprecipitation (ChIP) analyses to examine how depletion of DmGTSF1 would affect Piwi-piRISC targeting to TE loci on the genome. Piwi-RNAi and Mael-RNAi were carried out as control experiments (Sienski et al. 2012). Chromatin complexes were immunoprecipitated using anti-Pol II and anti-H3K9me3, from which DNA fragments were prepared and subjected to quantitative PCR (qPCR) for quantification of mdg1, gypsy, and roo elements in the complexes. Pol II accumulation to specific TEs and/or genes indicates derepression of the genomic elements (because they are transcribed by Pol II), whereas H3K9me3 accumulation indicates silencing ( $\mathrm{H} 3 \mathrm{~K} 9 \mathrm{me} 3$ is a silent chromatin marker). We found that a lack of DmGTSF1 resulted in accumulation of Pol II (Fig. 5A; Supplemental Fig. S7A) and less efficient enrichment of H3K9me3 (Fig. 5B) at $m d g 1$ and gypsy loci on the genome, although accumulation at roo loci was hardly affected. These results are consistent with the mRNA-seq data shown in Figure 3A. Thus, DmGTSF1 is required for PiwipiRISCs to regulate target TE loci on the genome. Depletion of DmGTSF1 did not change the cellular localization of Piwi (MycPiwi in Supplemental Fig S3D). Likewise, depletion of Piwi did not change the localization of DmGTSF1 (Myc-DmGTSF1 in Supplemental Fig S7B). We propose that DmGTSF1, which is a component of nuclear PiwipiRISCs, acts as a cofactor during Piwi-mediated transcriptional transposon silencing at the genomic sites where Piwi-piRISCs exert a silencing function.

Previous studies have shown that Piwi is imported to the nucleus only after being loaded with mature piRNAs at cytoplasmic $\mathrm{Yb}$ bodies in ovarian somas (Saito et al. 2010). In other words, cells use an elaborate system to avoid localization of Piwi to the nucleus when the protein is devoid of piRNAs. This "gatekeeping" regulation is crucial for control of cellular events and gonadal development because Piwi devoid of piRNAs in the nucleus would not know what to target. This study and an earlier one (Sienski et al. 2012) showed that two factors, Mael and DmGTSF1, are required for the targeting of PiwipiRISCs to achieve efficient TE silencing in the nucleus. Currently, it is unclear why this type of nuclear regulation is required and what functions of Mael and DmGTSF1 are required. To determine this, it will be important to isolate the nuclear Piwi-piRISC as a full unit, identify the components, and unveil their functions and the relationships among them. This would shed new light on nuclear RNA silencing pathways.

\section{Materials and methods}

Knockdown, Northern blotting, Western blotting, antibody production, immunoprecipitation, immunofluorescence, RNA sequencing (RNA-seq) analysis, plasmid construction, rescue assay, GST pull-down assay, and RT-qPCR analysis

siRNA transfection, Northern blotting, Western blotting, production of anti-DmGTSF1 antibody, immunoprecipitation, immunofluorescence, 
GST pull-down assay, and RT-qPCR analysis were performed as previously described (Supplemental Material; Saito et al. 2007, 2010). DNA oligonucleotides used in RT-qPCR are summarized in Supplemental Table S2. RNA-seq analysis, plasmid construction, and rescue assay are shown in the Supplemental Material.

\section{Drosophila strains and brooming analysis}

Yellow white $(\mathrm{y} w)$ and Oregon R were employed as wild-type strains. The DmGTSF1 allele used was $P\{w[+m C]=G S V 6\} G S 12962 / T M 3$ (Drosophila Genomics Resource Center stock no. 204406). All stocks were maintained at $25^{\circ} \mathrm{C}$. Mating tests were done in batches of test males and at least 10 virgin females per vial. The presence or absence of progeny was scored after at least $7 \mathrm{~d}$. For brooding tests, virgin $\mathrm{DmGTSF}^{+/-}$and DmGTSF1 ${ }^{-/-}$ females were mated to Oregon R males in yeasted apple agar plates. The females were left in the plate to continue to lay eggs until the 10th day after mating. Laid eggs on the plate were counted on the fourth, seventh, and 10th days under a microscope.

\section{ChIP assay}

Cells $\left(2 \times 10^{8}\right)$ were cross-linked with $1 \%$ formaldehyde for $10 \mathrm{~min}$ at room temperature, and cross-linking was then stopped with $125 \mathrm{mM}$ glycine for $5 \mathrm{~min}$ at room temperature. The DNA-protein complexes were lysed with $1 \%$ lysis buffer $(50 \mathrm{mM}$ HEPES-KOH at $\mathrm{pH} 7.4,150 \mathrm{mM}$ sodium chloride, $1 \mathrm{mM}$ EDTA, $1 \%$ Triton X-100, 0.1\% sodium deoxycholate, protease inhibitors). The cross-linked chromatin was sheared into $\sim 200$ base pairs using Covaris S2 ultrasonicators. The DNA-protein complexes were incubated with nonimmune IgG antibody, anti-Pol II antibody 8WG16 (Santa Cruz Biotechnology), or anti-H3K9me3 antibody (Abcam, 8898) immobilized on Dynabeads-Protein $\mathrm{G}$ for $2 \mathrm{~h}$ at $4^{\circ} \mathrm{C}$. The beads were washed with high-salt lysis buffer $(50 \mathrm{mM}$ HEPES-KOH at $\mathrm{pH}$ 7.4, $450 \mathrm{mM}$ sodium chloride, $1 \mathrm{mM}$ EDTA, 1\% Triton X-100, $0.1 \%$ sodium deoxycholate, protease inhibitors) and wash buffer $(50 \mathrm{mM}$ Tris- $\mathrm{HCl}$ at $\mathrm{pH}$ 8.0, $1 \mathrm{mM}$ EDTA, $250 \mathrm{mM}$ lithium chloride, 0.5\% NP-40, $0.5 \%$ sodium deoxycholate) followed by TE $(10 \mathrm{mM}$ Tris-HCl at $\mathrm{pH} 8.0,1 \mathrm{mM}$ EDTA). After reversing the cross-linking for $12-16 \mathrm{~h}$ at $65^{\circ} \mathrm{C}$, samples were treated with RNase A for $30 \mathrm{~min}$ at $37^{\circ} \mathrm{C}$, and then DNA was recovered. Short DNA fragments corresponding to retrotransposons were amplified using

A

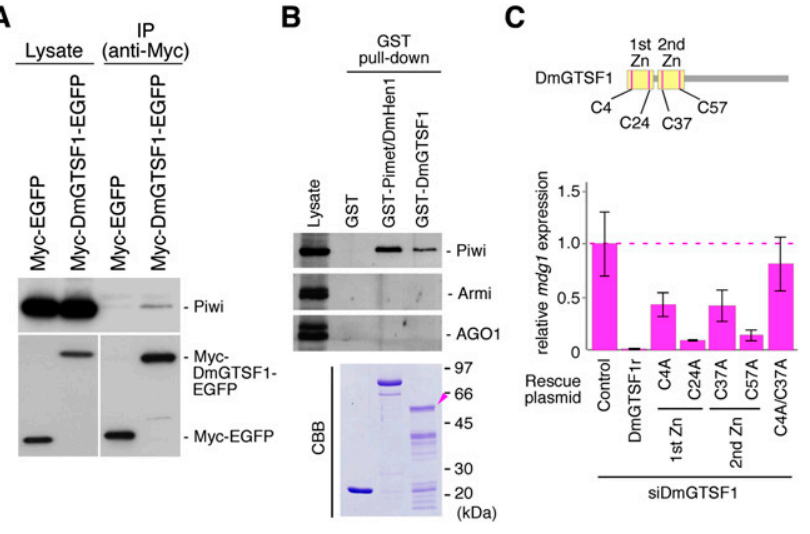

Figure 4. DmGTSF1 associates with Piwi in vivo and in vitro. $(A)$ Immunoprecipitation and Western blotting show that endogenous Piwi immunoprecipitated with Myc-DmGTSF1-EGFP but not with Myc-EGFP. (B) DmGTSF1 associates with PIWI but not with Armi or AGO1. Bound fractions with GST-Pimet/DmHenl and GST-DmGTSF1 were probed with antibodies against Piwi, Armi, and AGO1. (Bottom panel) An arrowhead shows where full-length GST-DmGTSF1 migrates on the Coomassie Brilliant Blue-stained gel. $(C$, top $)$ A schematic drawing of DmGTSF1. (Bottom) RNAi-resistant Myc-tagged full-length DmGTSFlr fused with EGFP (Myc-DmGTSF1r-EGFP) and $\mathrm{Zn}$ finger mutants were expressed in DmGTSF1-depleted OSCs. The expression levels of mdg1 were monitored by RT-qPCR. DmGTSF1 Zn finger mutants failed to rescue $m d g 1$ silencing lost following depletion of DmGTSF1 in OSCs.
A

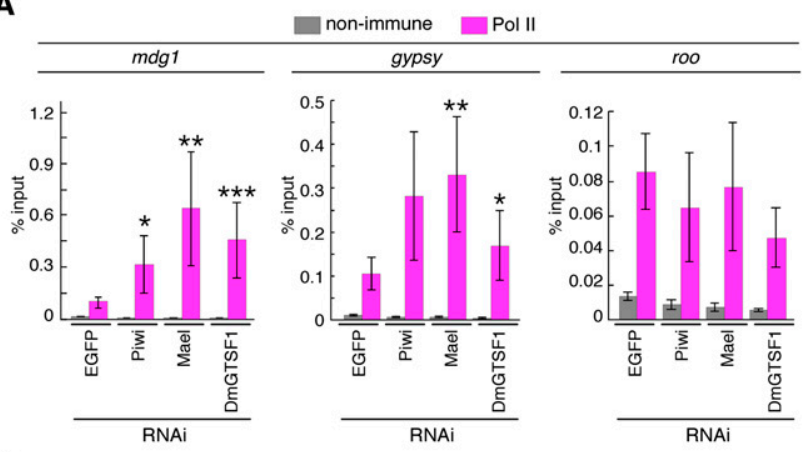

B

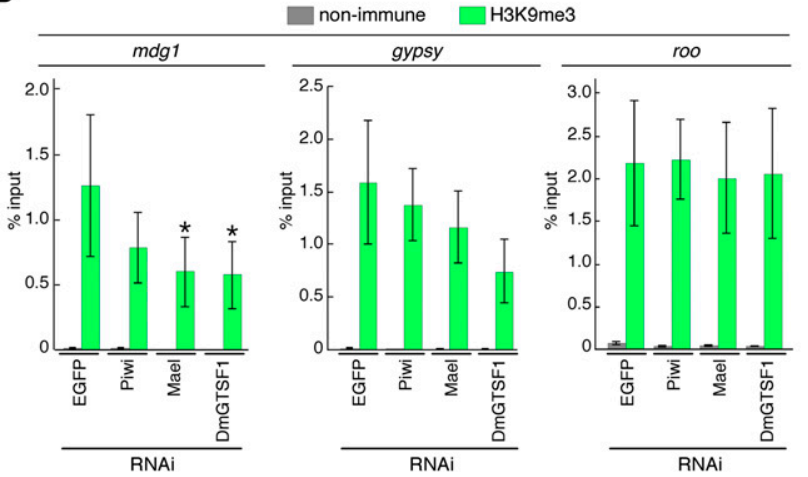

Figure 5. Depletion of DmGTSF1 increases the association of Pol II with retrotransposons and decreases the levels of $\mathrm{H} 3 \mathrm{~K} 9 \mathrm{me} 3$ on retrotransposons targeted by Piwi-piRISC. The associations of Pol II $(A)$ and H3K9me3 $(B)$ with $m d g 1$, gypsy, and roo were assayed by ChIP and qPCR. The results represent the percentage input values of three independent samples. Each experiment was repeated at least three times. Error bars indicate SEM values. $P$-values were calculated using the two-tailed Student's $t$-test for comparisons with control siRNAtransfected groups (EGFP). $\left(^{\star}\right) P<0.05$; $\left.^{\star \star}\right) P<0.01 \dot{1}^{\left({ }^{\star \star}\right)} P<0.001$.

a LightCycler 480 Real-Time PCR Instrument II with SYBR Premix Ex Taq (Takara). The primers used are listed in Supplemental Table S2. Pol II ChIP sequencing (ChIP-seq) libraries for EGFP, Piwi, Mael, and DmGTSF1 knockdown OSCs were analyzed using a single lane of Illumina MiSeq. This yielded $\sim 2.6$ million to 3.4 million genome-mapped reads in each transfected sample. For computational analyses, we trimmed for adaptor sequences, mapped the reads to Drosophila genome release $\mathrm{dm} 3$, and calculated reads per million genome mappers (RPM) values as described within the RNA-seq computational analysis section. Sequencing data are available through Gene Expression Omnibus (accession no. GSE47006).

\section{Acknowledgments}

We thank H. Ishizu, K. Sato, S. Yamanaka, and M. Kamatani for technical assistance, and other members of the Siomi laboratory for discussions and comments on the manuscript. We thank S. Inagaki for technical suggestions and comments regarding the immunofluorescence analyses. This work was supported by the Funding Program for Next-Generation WorldLeading Researchers (LS109) to K.S.; Core Research for Evolutional Science and Technology (CREST) from the Japan Society for the Promotion of Science and Technology (JSPS) to M.C.S.; a Grant-in-Aid for Scientific Research from the Ministry of Education, Culture, Sports, Science, and Technology (MEXT) of Japan to H.S. and M.C.S.; and a Grant-in-Aid for the Global COE program from MEXT to Keio University to H.S.

\section{Note added in proof}

While this manuscript was under revision, Muerdter et al. (2013) reported that DmGTSF1/CG3893 is required for TE silencing. The investigators termed DmGTSF1/CG3893 as Asterix (Muerdter et al. 2013). 


\section{References}

Andreeva A, Tidow H. 2008. A novel CHHC Zn-finger domain found in spliceosomal proteins and tRNA modifying enzymes. Bioinformatics 24: 2277-2280.

Aravin AA, Sachidanandam R, Bourc'his D, Schaefer C, Pezic D, Toth KF, Bestor T, Hannon GJ. 2008. A piRNA pathway primed by individual transposons is linked to de novo DNA methylation in mice. Mol Cell 31: 785-799.

Brennecke J, Aravin AA, Stark A, Dus M, Kellis M, Sachidanandam R, Hannon GJ. 2007. Discrete small RNA-generating loci as master regulators of transposon activity in Drosophila. Cell 128: 10891103.

Christerson LB, McKearin DM. 1994. orb is required for anteroposterior and dorsoventral patterning during Drosophila oogenesis. Genes Dev 8: $614-628$.

Darricarrère N, Liu N, Watanabe T, Lin H. 2013. Function of Piwi, a nuclear Piwi/Argonaute protein, is independent of its slicer activity. Proc Nat1 Acad Sci 110: 1297-1302.

Graveley BR, Brooks AN, Carlson JW, Duff MO, Landolin JM, Yang L, Artieri CG, van Baren MJ, Boley N, Booth BW, et al. 2011. The developmental transcriptome of Drosophila melanogaster. Nature 471: 473-479.

Haase AD, Fenoglio S, Muerdter F, Guzzardo PM, Czech B, Pappin DJ, Chen C, Gordon A, Hannon GJ. 2010. Probing the initiation and effector phases of the somatic piRNA pathway in Drosophila. Genes Dev 24: 2499-2504.

Horwich MD, Li C, Matranga C, Vagin V, Farley G, Wang P, Zamore PD. 2007. The Drosophila RNA methyltransferase, DmHen1, modifies germline piRNAs and single-stranded siRNAs in RISC. Curr Biol 17: 1265-1272.

Huang XA, Yin H, Sweeney S, Raha D, Snyder M, Lin H. 2013. A major epigenetic programming mechanism guided by piRNAs. Dev Cell 24: 502-516.

Ipsaro JJ, Haase AD, Knott SR, Joshua-Tor L, Hannon GJ. 2012. The structural biochemistry of Zucchini implicates it as a nuclease in piRNA biogenesis. Nature 491: 279-283.

Johnson E, Wayne S, Nagoshi R. 1995. $f_{S}$ (1) $Y b$ is required for ovary follicle cell differentiation in Drosophila melanogaster and has genetic interactions with the Notch group of neurogenic genes. Genetics 140: 207-217.

Karginov FV, Hannon GJ. 2010. The CRISPR system: Small RNA-guided defense in bacteria and archaea. Mol Cell 37: 7-19.

Ketting RF, Haverkamp TH, van Luenen HG, Plasterk RH. 1999. Mut-7 of C. elegans, required for transposon silencing and RNA interference, is a homolog of Werner syndrome helicase and RNaseD. Cell 99: 133-141.

Klenov MS, Sokolova OA, Yakushev EY, Stolyarenko AD, Mikhaleva EA, Lavrov SA, Gvozdev VA. 2011. Separation of stem cell maintenance and transposon silencing functions of Piwi protein. Proc Natl Acad Sci 108: 18760-18765.

Kuramochi-Miyagawa S, Watanabe T, Gotoh K, Totoki Y, Toyoda A, Ikawa M, Asada N, Kojima K, Yamaguchi Y, Ijiri TW, et al. 2008. DNA methylation of retrotransposon genes is regulated by Piwi family members MILI and MIWI2 in murine fetal testes. Genes DeV 22: 908-917.

Le Thomas A, Rogers AK, Webster A, Marinov GK, Liao SE, Perkins EM, Hur JK, Aravin AA, Toth KF. 2013. Piwi induces piRNA-guided transcriptional silencing and establishment of a repressive chromatin state. Genes Dev 27: 390-399.

Lim AK, Kai T. 2007. Unique germ-line organelle, nuage, functions to repress selfish genetic elements in Drosophila melanogaster. Proc Nat1 Acad Sci 104: 6714-6719.

Lin H, Spradling AC. 1997. A novel group of pumilio mutations affects the asymmetric division of germline stem cells in the Drosophila ovary. Development 124: 2463-2476.

Lynch M. 2007. The origins of genome architecture. Sinauer Associates, Sunderland, MA.

Muerdter F, Guzzardo PM, Gillis J, Luo Y, Yu Y, Chen C, Fekete R, Hannon GJ. 2013. A genome-wide RNAi screen draws a genetic framework for transposon control and primary piRNA biogenesis in Drosophila. Mol Cell 50: 736-748.

Nishimasu H, Ishizu H, Saito K, Fukuhara S, Kamatani MK, Bonnefond L, Matsumoto N, Nishizawa T, Nakanaga K, Aoki J, et al. 2012.
Structure and function of Zucchini endoribonuclease in piRNA biogenesis. Nature 491: 284-287.

Okamura K, Ishizuka A, Siomi H, Siomi MC. 2004. Distinct roles for Argonaute proteins in small RNA-directed RNA cleavage pathways. Genes Dev 18: 1655-1666.

Olivieri D, Sykora MM, Sachidanandam R, Mechtler K, Brennecke J. 2010. An in vivo RNAi assay identifies major genetic and cellular requirements for primary piRNA biogenesis in Drosophila. EMBO J 29: 3301-3317.

Pillai RS, Chuma S. 2012. piRNAs and their involvement in male germline development in mice. Dev Growth Differ 54: 78-92.

Rozhkov NV, Hammell M, Hannon GJ. 2013. Multiple roles for Piwi in silencing Drosophila transposons. Genes Dev 27: 400-412.

Ruohola H, Bremer KA, Baker D, Swedlow JR, Jan LY, Jan YN. 1991. Role of neurogenic genes in establishment of follicle cell fate and oocyte polarity during oogenesis in Drosophila. Cell 66: 433-449.

Saito K, Sakaguchi Y, Suzuki T, Siomi H, Siomi MC. 2007. Pimet, the Drosophila homolog of HEN1, mediates 2'-O-methylation of Piwiinteracting RNAs at their 3' ends. Genes Dev 21: 1603-1608.

Saito K, Inagaki S, Mituyama T, Kawamura Y, Ono Y, Sakota E, Kotani H, Asai K, Siomi H, Siomi MC. 2009. A regulatory circuit for piwi by the large Maf gene traffic jam in Drosophila. Nature 461: 1296-1299.

Saito K, Ishizu H, Komai M, Kotani H, Kawamura Y, Nishida KM, Siomi $\mathrm{H}$, Siomi MC. 2010. Roles for the $\mathrm{Yb}$ body components Armitage and $\mathrm{Yb}$ in primary piRNA biogenesis in Drosophila. Genes Dev 24: 2493-2498.

Sato K, Nishida KM, Shibuya A, Siomi MC, Siomi H. 2011. Maelstrom coordinates microtubule organization during Drosophila oogenesis through interaction with components of the MTOC. Genes Dev 25: 2361-2373.

Senti KA, Brennecke J. 2010. The piRNA pathway: A fly's perspective on the guardian of the genome. Trends Genet 26: 499-509.

Sienski G, Donertas D, Brennecke J. 2012. Transcriptional silencing of transposons by Piwi and maelstrom and its impact on chromatin state and gene expression. Cell 151: 964-980.

Siomi H, Siomi MC. 2009. On the road to reading the RNA-interference code. Nature 457: 396-404.

Siomi MC, Sato K, Pezic D, Aravin AA. 2011. PIWI-interacting small RNAs: The vanguard of genome defence. Nat Rev Mol Cell Biol 12: 246-258.

Slotkin RK, Martienssen R. 2007. Transposable elements and the epigenetic regulation of the genome. Nat Rev Genet 8: 272-285.

Szakmary A, Reedy M, Qi H, Lin H. 2009. The Yb protein defines a novel organelle and regulates male germline stem cell self-renewal in Drosophila melanogaster. J Cell Biol 185: 613-627.

Tabara H, Sarkissian M, Kelly WG, Fleenor J, Grishok A, Timmons L, Fire A, Mello CC. 1999. The rde-1 gene, RNA interference, and transposon silencing in C. elegans. Cell 99: 123-132.

Wang SH, Elgin SC. 2011. Drosophila Piwi functions downstream of piRNA production mediating a chromatin-based transposon silencing mechanism in female germ line. Proc Natl Acad Sci 108: 21164-21169.

Yoshimura T, Miyazaki T, Toyoda S, Miyazaki S, Tashiro F, Yamato E, Miyazaki J. 2007. Gene expression pattern of Cue110: A member of the uncharacterized UPF0224 gene family preferentially expressed in germ cells. Gene Expr Patterns 8: 27-35.

Yoshimura T, Toyoda S, Kuramochi-Miyagawa S, Miyazaki T, Miyazaki S, Tashiro F, Yamato E, Nakano T, Miyazaki J. 2009. Gtsf1/Cue110, a gene encoding a protein with two copies of a CHHC Zn-finger motif, is involved in spermatogenesis and retrotransposon suppression in murine testes. Dev Biol 335: 216-227. 


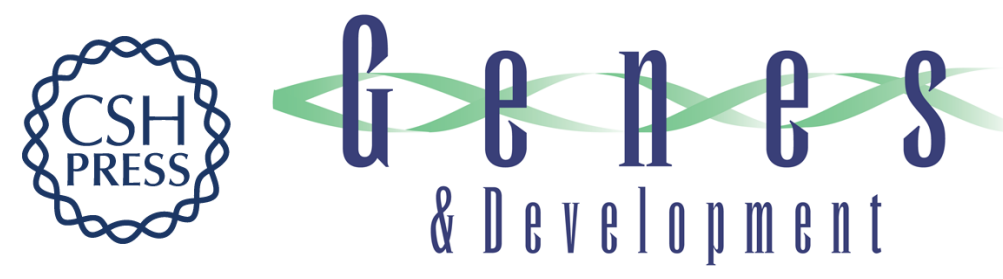

\section{DmGTSF1 is necessary for Piwi-piRISC-mediated transcriptional transposon silencing in the Drosophila ovary}

Hitoshi Ohtani, Yuka W. Iwasaki, Aoi Shibuya, et al.

Genes Dev. 2013, 27:

Access the most recent version at doi:10.1101/gad.221515.113

Supplemental http://genesdev.cshlp.org/content/suppl/2013/08/02/27.15.1656.DC1
Material

References This article cites 39 articles, 16 of which can be accessed free at:

http://genesdev.cshlp.org/content/27/15/1656.full.html\#ref-list-1

License

Email Alerting

Receive free email alerts when new articles cite this article - sign up in the box at the top

Service

right corner of the article or click here.

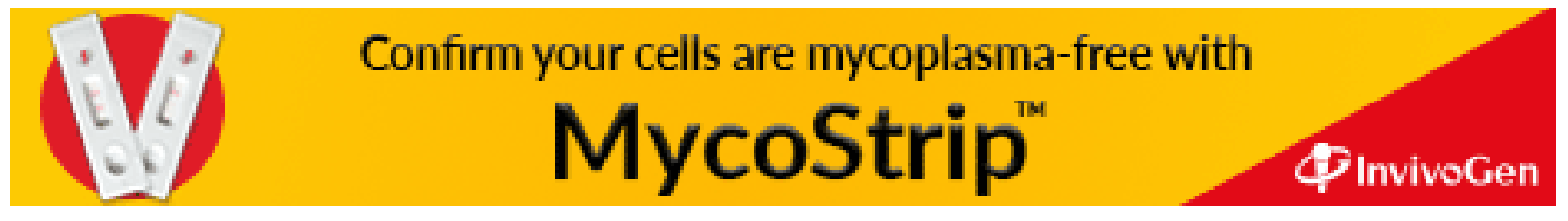

